\title{
A practical clinical course model for use during the COVID- 19 pandemic
}

\author{
Tan, Y.J. and Manohararaj, N.
}

\begin{abstract}
The coronavirus disease (COVID-19) pandemic brought unprecedented challenges to and detrimental effects on medical training and education. Due to existing cordon sanitaire measures aimed at containing the infection, clinical courses and professional examinations involving physical contact between participants and with patients have been repeatedly cancelled or postponed. Herein, we describe a tried-and-tested clinical course model designed to lower the risks of transmission between and beyond the participants, whilst remaining practical and pragmatic in its structure, so as to allow the implementation by academic medical centres worldwide during the current and future pandemics.
\end{abstract}

Keywords: COVID-19, Pandemic, Medical education, Postgraduate, MRCP, Singapore

\section{Background}

The coronavirus disease 2019 (COVID- 19) pandemic nears its first year, inflicting havoc across the global health systems, seemingly with no end in sight. Medical education and training did not escape unscathed, as vital healthcare resources are channeled toward the control of the outbreak, medical training was relegated to a lower priority - suspension of clinical rotations, postponement of clinical tutorials, and the cancellation or modification of the undergraduate aptitude in clinical examination, interview and counselling, and passing PACES, and thus passing MRCP, remains a mandatory criterion for one to graduate from Singapore's Internal Medicine Residency Programme (MOH, 2020). and postgraduate examinations (Liang et al., 2020). The United Kingdom's (UK) Membership of the Royal Colleges of Physicians assesses the trainees' preparing for PACES amidst the pandemic was difficult, with mushrooming clinical workload reducing the time available for both the residents and tutors to learn and to teach respectively (Liang et al., 2020).

National Neuroscience Institute, Singapore

Corresponding author: Dr. Tan You Jiang

Whyjae@gmail.com

DOI: http://doi.org/10.4038/seajme.v15i2.270
(MRCP) Practical Assessment of Clinical Examination Skills (PACES) examination was no exception (MRCPUK, 2020). PACES As COVID-19 raged across the globe, repeated cancellations of PACES examinations in early 2020 resulted in a backlog of residents who were at risk of being unable to graduate. Preparations were further confounded by the suspension of clinical rotations and the restriction of movement within the hospitals, limiting the variety of patients available for the residents to examine. Recognizing the ostensible need for a PACES preparatory course, our Academic Medical Centre (AMC) designed a neurology clinical examination course while stringently adhering to prevailing infection control measures, allowing our residents to effectively and safely learn amidst the global pandemic.

\section{Activity}

Designing a clinical course amidst the COVID19 pandemic

Being the first clinical course in Singapore in 2020 after a partial de-escalation of national cordon sanitaire measures, we were without a precedent. Adherence to the recommendations 
described within the Singapore's Ministry of Health's guidelines (Confidential circular: 195/2020) was mandatory:

1. Lowering the risks of transmission between the participants (i.e. trainees, patients, tutors and administrative team members)

2. Lowering the risks of transmission beyond the participants (i.e. their families and colleagues etc.)

\section{Facilitation of contact-tracing}

We designed our course around these recommendations, while weaving into its design the flexibility necessary for modification and implementation by other academic medical centres (AMCs) in future clinical courses.

Selection of patients, trainees, tutors and administrative team members

Six patients were chosen based on their respective neurologic conditions' teaching points. Patients who visited or were due to visit a medical facility within two weeks before or after the course were excluded. Only tutors, trainees and administrative staff from our AMC were eligible to participate. The number of candidates was capped at twelve, so as to create a low trainees-to-tutor ratio of five to one. These was intended to reduce the risk of COVID-19 transmission within and without $\mathrm{SGH}$, and to facilitate contact-tracing efforts should an infection occur.

\section{Pre-course preparations and measures}

The administrative team screened all participants screened for fevers, respiratory symptoms, significant travel and contact history via telephony during the week before the course. Their contact numbers and addresses were collected to facilitate contract tracing when required.

\section{Registration processes at the venue}

The course took place in a facility detached from our clinical areas. All participants (patients, tutors, trainees and the administrative team) were required to register on the
SafeEntry system, a national digital check-in system that logs the identity and mobile numbers of individuals when visiting a particular public venue, designed to augment the contact tracing mechanisms and to identify COVID-19 clusters (SafeEntry, 2020). They were also required to physically register at the reception, during which their surface temperatures were measured with an infrared thermometer, and their addresses and contact numbers were reaffirmed and recorded. A questionnaire was administered on all participants to screen for fevers, respiratory symptoms, and significant travel and contact history. Participants who screened positive were excluded from the course.

\section{Deployment and disposition of the patients}

Acknowledging the risks of COVID-19 transmission, the six patients were divided into two consecutive sessions of three patients each. The course begins with three patients in the first session, followed by the next three in the second. During each session, the three patients were separately held in their assigned examination rooms and were disallowed from interacting with each other. Patients from the first session will individually depart 30 minutes before arrival of the next three patients. Our measures ensured zero contact between the patients during the entire course.

Grouping of the trainees and tutors, and the implementation of infection control measures

Twelve trainees were divided into three groups of four. A tutor was attached to each group throughout the entire course (trainee-tutor ratio of $1: 4)$. The three groups are kept separated throughout, with their constituent trainees and tutors moving together between patients (figure 1). Each group were given 45 minutes per patient, allowing ample time for clinical examination, presentation, discussion and teaching. By fixing a single tutor to a small group throughout the entire course, we intended to emulate the museum's 'guided tours' model, in which the visitors are informatively and comfortably guided through collections of masterpieces. This, coupled with the group's small size, permitted closer 
supervision and interactive guidance, creating a learning climate in which the trainees received immediate feedback, and were encouraged to question and clarify without fear (Lama et al., 2015).

Importantly, the small size also allowed for social distancing measures to be implemented with ease (Ryu et al., 2020). Trainees who were not examining the patient were kept socially distanced at least 1 meter apart from each other. Surgical masks were worn by all (throughout the course, except when clinical examination required its removal (e.g. examination of the cranial nerves). Hand hygiene was practiced using alcohol hand-rub (100\% ethanol) before and after contact with the patient and his/her surroundings. Prior to leaving, each patient received a memorandum which detailed their participation in the course, and included our contact information. Patients were instructed to provide the memorandum to their treating doctor should they present to a medical facility due to illness. The examination rooms and the clinical equipment were thoroughly disinfected with cleaning wipes (94\% ethanol) after each session.

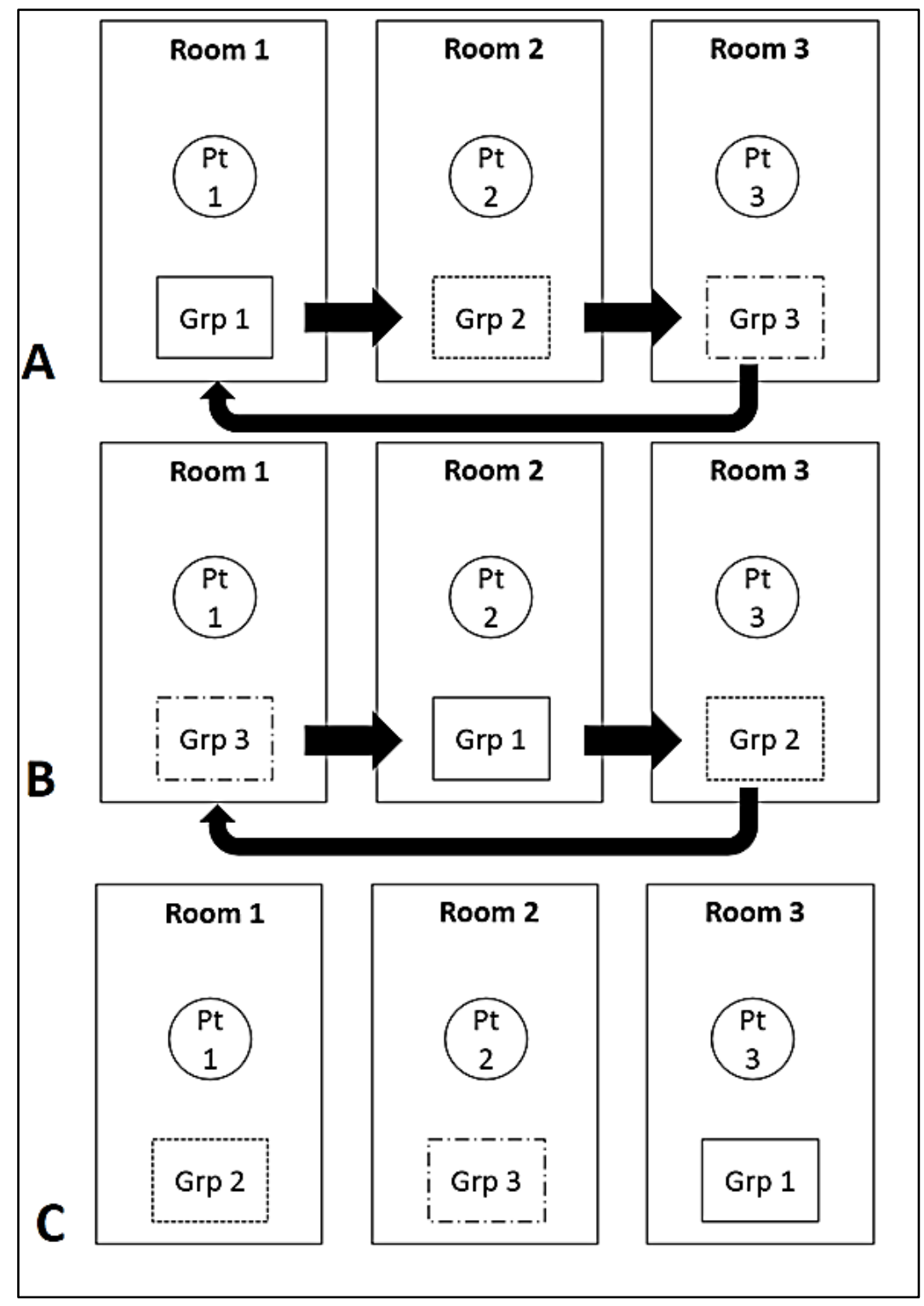

Figure 1: Graphical representation of the first session of our course.

The patients are kept within their assigned rooms, while the groups rotate through all three rooms $(A, B$ and $C$ in sequential order). There is no physical contact between the patients and between the groups. The second session uses an identical format. Abbreviations: Grp, group; Pt, patient. 


\section{Results and Discussion}

The COVID-19 pandemic has presented unique challenges for medical education. Despite the prevailing limitations, a clinical course can be effectively and safely held whilst adhering to infection control measures. Simplicity and flexibility were deliberately interwoven into our course design, so that it may be easily adopted and implemented by other AMCs during this pandemic:

- Low manpower requirement: only 1 administrative staff and 3 tutors were required for this course

- The course's layout can be easily replicated in most buildings

- No special equipment was required. The alcohol hand rubs and swabs used are internationally available and easily obtainable.

- The size and composition of the 'smallgroups' can be easily altered in accordance to the prevailing nation infection control measures of the host AMC.

Our course's design provided a simple framework for implementation by other AMCs amidst the current COVID-19 crisis, and as such has significant potential in reducing further interruptions to clinical training, as future pandemics appear increasingly inevitable.

\section{Compliance with Ethical Standards}

Funding: None

Conflicts of interest: None

Ethical approval: NA

Informed consent: NA

\section{References}

Lama, P., Kulkarni, J., Tamang, B.K., Sinha, P., 2015. The impact and significance of small and large group teaching and learning in medical curriculum. SMU Med J. 2(2):175-82.

Liang, Z. C., Ooi, S., \& Wang, W., 2020. Pandemics and Their Impact on Medical Training: Lessons from Singapore. Academic medicine: journal of the Association of American Medical Colleges, 95(9), 1359-1361.

MRCPUK. MRCP (UK) and specialty examinations cancelled until September 2020. https://www.mrcpuk.org/news/mrcpuk-andspecialty-certificate-examinations-cancelleduntil-september-2020. Accessed November 01, 2020.

$\mathrm{MOH}$ (Ministry of Health, Singapore). Internal Medicine Examination https://www.healthprofessionals.gov.sg/sab /specialist-examination/internal-medicineexamination-(2020)/overview. Accessed November 01, 2020.

Ryu, S., Gao, H., Wong, J. Y., Shiu, E., Xiao, J., Fong, M. W., \& Cowling, B. J., 2020. Nonpharmaceutical Measures for Pandemic Influenza in Non healthcare SettingsInternational Travel-Related Measures. Emerging infectious diseases, 26(5), 961-966.

SafeEntry. https://www.safeentry.gov.sg/. Accessed November 11, 2020. 